\title{
Decision Making in Evolutionary Optimization (Abstract of Invited Talk)
}

\author{
Carlos M. Fonseca \\ Universidade do Algarve \\ Campus de Gambelas, 8005-139 Faro, Portugal \\ cmfonsec@ualg.pt
}

\begin{abstract}
Current evolutionary multiobjective optimization (EMO) approaches tend to emphasize the approximation of the Pareto-optimal front as a whole, thereby dissociating the optimization process from the selection of the final compromise solution by a decision maker. This has the advantage of removing subjective preference information from the optimization problem formulation, but it also makes the resulting problem computationally more demanding. In order to concentrate the search effort on the regions of potential interest to the decision maker, techniques for the progressive articulation of preferences in EMO have been proposed, casting EMO as the interaction between an evolutionary search mechanism and a decision maker. It is worth noting that even the promotion of diversity across the Pareto-optimal front, which is generally regarded as an optimizer design issue, may be successfully addressed by the decision maker within this framework, as it has been proposed recently by others. Regarding the evolutionary search mechanism, the main question at each iteration consists of determining the next candidate solution(s) to be evaluated, given the information acquired since the beginning of the run. This may be seen as another decision-making problem, but one with (very) incomplete attribute information, since objective values are generally not known for most potential alternatives. Alternatively, it may be seen as a control problem, where actions (new solutions) are to be selected based on the feedback provided by the decision maker. Either way, some model, however weak, of the underlying optimization problem must be assumed. In this talk, both the evaluation of current solutions and the generation of new candidate solutions in EMO will be discussed from a decision making perspective. From the discussion, opportunities for incorporating more explicit decision making in EMO will be identified.
\end{abstract}

